\title{
Evolutionary trends in directional hearing
}

Dr. Catherine E. Carr

Phone: 1-301-405-6915

Email: cecarr@umd.edu

Department of Biology,

University of Maryland

College Park MD, USA

Dr. Jakob Christensen-Dalsgaard

Phone: 45-6550-2448

Department of Biology,

University of Southern Denmark

Campusvej 55

5230 Odense M, Denmark 


\begin{abstract}
Tympanic hearing is a true evolutionary novelty that arose in parallel within early tetrapods. We propose that in these tetrapods, selection for sound localization in air acted upon pre-existing directionally sensitive brainstem circuits, similar to those in fishes. Auditory circuits in birds and lizards resemble this ancestral, directionally sensitive framework. Despite this anatomically similarity, coding of sound source location differs between birds and lizards. In birds, brainstem circuits compute sound location from interaural cues. Lizards, however, have coupled ears, and do not need to compute source location in the brain. Thus their neural processing of sound direction differs, although all show mechanisms for enhancing sound source directionality. Comparisons with mammals reveal similarly complex interactions between coding strategies and evolutionary history.
\end{abstract}

\title{
Text
}

The addition of new sensory systems provides a unique challenge for the brain and a window into how it responds to evolutionary change [1]. We focus here on changes in the auditory system associated with the addition of new ears, after tetrapods emerged onto land. Predation makes sound source localization evolutionarily important [2], and how animals evolved to hear airborne sound has been an open question.

Several recent papers suggest that the first terrestrial tetrapods could have had similar hearing to modern lungfish, and thus some sensitivity to low frequency airborne sound [3], even though they lacked middle ear adaptations for hearing in air. Like lungfish, early aquatic tetrapods could probably hear under water by sensing the particle motion component of sound $[3,4]$. In air, air breathing and the resonance of air in the lungs could have conferred sensitivity to sound pressure, although lungfish do not have specialized connections between lungs and ears. Finally, even though lungfish lack a middle ear, they can detect airborne sound through detection of sound-induced head vibrations [3].

Recent amphibians, such as salamanders, provide models for hearing at a later stage of evolution, where patches of hair cells without an otoconial covering were formed. Both terrestrial adults and aquatic juvenile salamanders can detect airborne sound [5] with lower thresholds and at higher frequencies than lungfish. Collectively, these findings suggest that early atympanic tetrapods may have been pre-equipped for hearing in air [6].

\section{Tympanic hearing is a true evolutionary novelty}

The emergence of thin flexible tympana would have greatly increased the frequency range and sensitivity of hearing in air [7]. Tympana are not primitive to tetrapods, but instead the fossil record shows that the bony elements that support the tympanum appeared independently in the anurans, turtles, lepidosaurs, archosaurs, and mammals sometime in the Triassic, or about 120 Mya after the emergence of tetrapods [6,8]. Thus, sensitive high frequency hearing evolved multiple times, in parallel.

A pivotal new paper by Kitazawa et al. [9] confirms the parallel evolution of eardrums in at least two tetrapod lineages by showing that the tympanum forms in different ways in 
mouse and chicken. Tracing the formation of the jaw showed that the lower-to-upper jaw transformation induced by inactivation of the Endothelin1-Dlx5/6 cascade involving Goosecoid results in loss of the tympanic membrane in mouse, but causes duplication of the tympanic membrane in chicken [9]. Different development of the jaw joint and first pharyngeal pouch, perhaps related to feeding or breathing strategies, could have led to the coupling of tympanic membrane formation to the lower jaw in mammals and to the upper jaw in birds (Figure 1).

The emergence of the tympanic ear would have provided the previously insensitive, low frequency auditory system with increased sensitivity to sound and a wider frequency range, and led to changes in the central auditory system [10].

\section{Selection for hearing must act upon an existing octaval framework}

In all vertebrates, first order medullary nuclei receive octaval input and project to the sensory midbrain [11]. The eighth nerve is part of the octavolateralis system (octaval = ear with hair cells; lateralis = external lateral line with hair cells). Although neighbors, the eighth, lateral line and electrosensory cranial nerves are distinct, with the eighth nerve originating from an otic placode separate from the adjacent lateral line and electrosensory placodes [12]. Electroreception is an ancient division of the lateral line sensory system and ampullary electroreceptors are derived from lateral line placodes [13]. Electroreceptors were lost in the lineages that led to frogs, amniotes, and most rayfinned fishes.

In fishes, eighth nerve fibers enter the hindbrain and divide to synapse on a column of first order neurons in the dorsolateral medulla [14,15]. Four nuclei (five in telosts) are arranged rostro-caudally in the octaval column: anterior, descending, magnocellular, and posterior (Figure 1a). None of the nuclei are solely auditory, and only the anterior and descending octaval nuclei contain neurons that project to the auditory midbrain, forming the ascending auditory pathway. Auditory and vestibular systems are segregated to some extent in these nuclei, with auditory responses more dorsal than vestibular [16]. We assume that the first order projections in tetrapods are in some way derived from this octaval column, but there is substantial anatomical diversity among the major vertebrate taxa, and no single auditory nucleus below the midbrain can be agreed to be homologous across vertebrates $[17,18]$, perhaps in part because the new tympana exerted different effects, possibly due to their different embryological origins and location.

How could the new tympana exert an organizing effect on the inner ear and CNS? In the inner ear, the sensitivity to higher frequencies could have led to elongation of the auditory papillae in order to accommodate a wider frequency range. Genetic changes would have been needed [1], but at present, very little is known about the guidance cues that lead auditory and vestibular nerve fibers to their targets [19]. Eph receptors and their ligands are expressed in chicken eighth nerve, and this signaling pathway may play a role in target selection and the emergence of topographic maps of the developing auditory projections [19]. Potentially, Eph proteins could act to sort axons to their correct central locations for auditory and vestibular targets, since their disruption alters the spatial allocation of auditory versus vestibular target areas in the brainstem [20].

Other recent findings have begun to constrain hypotheses about the origins of the first order auditory nuclei and to lend support to the hypothesis that a variety of derivatives of the octaval column, and perhaps other rhombic lip regions, formed the first order nuclei of the tetrapod central auditory system [21]. In 1995, a pioneering chick-quail chimera 
study revealed the rhombomeric origins of the first order auditory nuclei [22,23]. Results from genetic fate mapping studies of mouse cochlear nucleus did not entirely agree with the results of the chick quail chimera study [24], which is now less surprising given Kitazawa's study of the origins of the tympanum. Mouse first order auditory nuclei form as genetically separable progenitor populations in "rostrocaudal neuromeric microdomains" around the hindbrain rhombic lip. The dorsal cochlear nucleus, which is only found in mammals, and is similar to the cerebellum [25], arises from microdomains caudal to and noncontiguous to cerebellar primordium, while the ventral cochlear nuclei arise from more rostral regions [24,26](Fig. 1b). The recent availability of genomic sequences from all major vertebrate taxa provide an unprecedented opportunity to investigate development and evolution of the auditory hindbrain [27,28],

These findings provide some idea of how the first order auditory nuclei might have been organized. Next we will consider how the neural coding of sound source location might have emerged.

\section{Neural processing of sound source location}

\section{Particle motion is directional}

The earliest form of localization relied on the detection of the direction of particle motion generated by sound sources, and it is reasonable to suppose that the first tetrapods were sensitive to this cue. Particle motion is easily transmitted through the fish's soft tissues to the endorgans of the ear because of the similar density of tissue and water. In the endorgans, the sensory hair cells are organized in a variety of orientations that provide the ear with the ability to respond to sounds originating from different directions. Sensory epithelia generally contain a stereotyped alignment of physiologically polarized hair cells [29] that provide inherent sensitivity to sound source direction, which is encoded by phase-locked activity in auditory afferents [16]. Underwater, a sound source from a particular direction will maximally excite the sensory hair cells with the same orientation, and excitation from other directions will fall off proportionally with a null perpendicular to the best axis. Other populations with different orientations have different best directions, and comparisons between different orientations allow disambiguation of sound source intensity and direction. These comparisons could be "monaural", i.e., between first order neurons receiving input from hair cells with different best orientations from the same sensory epithelium, or "binaural", between brainstem neurons receiving inputs from left and right epithelia. Monaural comparisons of onset responses from oppositely oriented hair cells within the epithelium might resolve the $180^{\circ}$ ambiguity in particle velocity responses (caused by particle oscillation around a rest position). However, sensitivity to sound pressure and binaural comparisons may sharpen the directional response and produce lateralization cues by comparisons of the responses of the two ears, since the best directions for the left and right ear differ sufficiently to allow azimuthal comparisons [30]. Recordings from the toadfish have shown a sharpening of the directional response in both the first order descending nucleus and in the midbrain torus [16]. Thus most fishes possess the ability to determine sound source direction.

In consequence, it is highly likely that the early atympanate tetrapods would have possessed neural circuits sensitive to sound source direction in water, although their different hair cell orientations would have not been as useful in air. Directional sources in air could have included substrate sound/bone conduction, i.e. sound-induced vibrations of the skull and body. It is possible that in these ancestral tetrapods, low-frequency sound may have been processed by non-tympanic mechanisms like those in extant amphibians. Extratympanic sensitivity in frogs produces a directional response in low- 
frequency auditory neurons with equally strong responses from ipsi- and contra-lateral directions, but with an almost 180 degree phase shift between ipsi- and contra-lateral stimulation [31]. Binaural EI neurons, like those found in toadfish, could transform and sharpen such a response by suppression [16].

\section{Coupled tympanic ears are inherently directional}

Everything changed with the evolution of tympanic hearing, which would have altered both the frequency response and sensitivity of the ears. When the tympana evolved, the left and right ears in some of the groups would have been acoustically coupled through the mouth cavity $[7,8]$. Coupled ears are inherently directional, acting as pressure difference receivers $[32,33]$. This is because sound can reach both the external and the internal surface (through the contralateral eardrum and mouth cavity). Depending on the phase difference of sound at the external and internal surface, eardrum motion will be increased or decreased by this binaural interaction. The output of the coupled ears also generates a much larger internal delay than that predicted from the arrival-time delay at the eardrums [34]. This is most clearly seen in modern lizards, where recordings from the auditory nerve reveal highly directional responses. Lizard auditory nerve responses are sensitive to both interaural time differences (ITD) and interaural level differences (ILD), reflecting the acoustical interactions of direct and indirect sound components at the eardrum (Fig. 2a)[34]. The coupling of the tympana means that all or almost all auditory responses in the lizard's central auditory system should be directional, without a requirement for computation of sound source location in the central nervous system. Animals with coupled ears still require binaural comparisons to differentiate between loud sounds at "poor" locations, where their tympanum is less sensitive, and quiet sounds at "good" locations. This is a very similar situation to fish that are sensitive to particle motion, where auditory nerve fibers also have inherently directional responses $[16,35]$.

Given the similarities in the nature of the directional information, it is possible that the existing auditory circuits in early atympanate tetrapods could have been repurposed to process directional sound in air. In the toadfish, auditory information projects to the descending and anterior octaval nuclei, both of which project to the auditory midbrain, although the descending nucleus projection is more prominent. The descending nucleus also projects within the medulla, with ipsilateral projections within the descending nucleus, and secondary projections to the contralateral descending nucleus [16]. These connections are very similar to what is currently known about diapsid auditory circuits, where the first order nucleus magnocellularis projects bilaterally to the adjacent nucleus laminaris in lizards [36], crocodilians and birds [17], and the nucleus laminaris projects to the auditory midbrain (Fig. 2). A scenario for the formation of the diapsid auditory circuits could be one where the ancestral descending nucleus was separated from its more ventral, vestibular neighbor, and then itself divided into first and second order nuclei, with the second order nucleus projecting to the midbrain (Figure 1c). The anterior nucleus resembles the nucleus angularis, and a similar parcellation into auditory and vestibular components would be required of it.

\section{Uncoupled tympanic ears and a need for central computation of sound source location}

There is no evidence that mammal ancestors ever had an open middle ear cavity. Their middle ears arose de-novo, as did their Eustachian tubes [9,37], and thus mammals may have always had a requirement for neural computation of directionality. This is not 
unique; in some of the archosaurs, most notably the barn owl, the frequencies of interest have shifted to frequencies where the ear coupling is insignificant [38], creating similar selection pressures for neural computation.

Sharpening and disambiguating directional inputs is computationally very different from de novo computation of binaural cues. Localization of sound sources with uncoupled ears relies on specialized pathways for detection of ITD and ILD. The ITD cues are represented by phase locked responses in the auditory nerve and are limited to frequencies below $2 \mathrm{kHz}$ in mammals and $9 \mathrm{kHz}$ in barn owls, whereas ILD cues are generated by sound diffraction by the body of the animal and generally limited to higher frequencies [39] and processed in a specialized nucleus, the lateral superior olive. ITD computations are first carried out in tonotopically-organized areas of the brainstem nucleus laminaris in birds and the medial superior olive in mammals (Fig. 2b,c). In birds, the ITD detection circuit conforms to the Jeffress or place model, where the first order nucleus magnocellularis encodes stimulus timing and projects bilaterally to the nucleus laminaris, whose neurons act as coincidence detectors [40]. A defining feature of the Jeffress model is the systematic representation of ITDs, creating a map of azimuthal position. In this scheme, individual neurons have different best ITDs, and all locations are represented by a labeled line or place code (Fig. 2b). Labeled lines are well suited to encode continuous variables like ITDs, and could be visualized as an array in the brain [40]. There is support for maps of ITD in barn owls [41,42] and chickens [43,44]. Data from small mammals, however, are inconsistent with maps of ITD [45]. Instead, the two medial superior olives may contain broadly tuned populations of neurons that represent ITD via firing rate, and where comparison of the two populations, or channels, may be used to decode ITD (the 'meter' strategy, Fig. 2c).

Do the map and meter coding strategies reflect evolutionary history or optimal coding? Potentially this is a false dichotomy, since experimental focus on differentiating between the two strategies has revealed not one but many solutions to encoding ITD. Recent comparisons of five mammalian species with a range of head sizes, from macaques to gerbils, suggest ITD representations in mammals may depend on head size and sound frequency [46]. Since most studies of ITD coding in mammals are from low frequency $(<1,500 \mathrm{~Hz})$ medial superior olive recordings, and most avian studies are from barn owls, which are specialized for high frequency ITD, it is difficult to distinguish between adaptations due to ancestry and frequency-related adaptations. This led Palanca-Castan and Köppl [42] to record from the low-frequency regions of nucleus laminaris of the barn owl, revealing data consistent with maps of ITD (Fig. 2b). Thus, some differences in ITD coding strategies may reflect evolutionary differences [47].

In sum, the interactions between coding strategies and evolutionary history are complex. Palanca-Castan and Köppl also compared low-frequency ITD coding in chicken [44] and found support for a modified place map that uses the slopes of ITD-selective response functions, instead of their peaks, to topographically represent ITD at mid- to high frequencies. At low frequencies below several $100 \mathrm{~Hz}$, their data did not support any current models of ITD coding. This is different to the nucleus laminaris in the predatory, accurate barn owls and suggests that constraints on optimal ITD processing could be related to the animal's ecological niche [44]. With respect to environmental constraints, high frequency hearing predominates in many mammals, and it has even been proposed that ITD processing in medial superior olive could be derived from an original ILD processing circuit, in a scenario where early mammals were small, possibly nocturnal, and more dependent upon processing of higher frequency sounds [48]. A focus on environmental constraints fits with the finding that localization abilities in mammals are 
correlated with the ability to direct the field of best vision to a sound source [49].

\section{Acknowledgements}

We gratefully acknowledge helpful insights from Drs. Shigeru Kuratani, Geoff Manley, Luis Puelles and Peggy Edds-Walton and assistance from G. Capshaw. This research was sponsored by National Institute on Deafness and Other Communications Disorders (NIDCD) grant DC-000436 (CEC) and by the Danish Natural Science Research Council (J.C-D). 


\section{Legends}

Figure 1. Proposed scenario for formation of the first order auditory nuclei after evolution of the tympanic membrane and middle ear.

Lateral views of the brainstem first order auditory nuclei [14] aligned with scenarios for separate formation of the tympanic membrane and middle ear in mammals and diapsids from [9]. Diagrams show the split between bony and lobe-finned fish (sarcopterygians), with a holostean as an example of bony fish that has retained basal characteristics, and mammals and diapsids as examples of sarcopterygian descendants.

Above: (a) A lateral view of the brainstem of the holostean, Amia calva, with the positions of all first order octavolateralis nuclei projected onto the lateral surface of the brain. Anterior (AON), Magnocellular (MON), Descending (DON) and Posterior (PON). The auditory targets are shown in blue above vestibular nuclei (grey). The cerebellar crest is shown above the octaval column (pale blue, grey dotted line). Modified from [14]. (b) Mammalian pattern in the same lateral view as in Amia, but with the mammalian ventral cochlear nucleus (VCN, blue) and dorsal cochlear nucleus shown above the vestibular column (DCN, pale blue cerebellar like structure plus blue first order auditory).

(c) Diapsid pattern with nucleus angularis (NA), nucleus laminaris (NL) and nucleus magnocellularis (NM) in blue above the vestibular column.

Below: Hypothetical scenario for tympanic membrane formation in mammals and diapsids modified from [9]. Purple and green structures represent the lower and upper jaw elements in an amniote ancestor where the primary jaw joint was distant from the first pharyngeal pouch. (a) Jaw structures in embryonic Amia showing Meckel's cartilage, the pteroquadrate $(\mathrm{q})$ and hyomandibular $(\mathrm{h})$ perforated by a branch of the facial nerve, modified from [50]. (b) In mammalian ancestors, the primary jaw joint moved dorsal, associating the lower jaw with the tympanum and tympanic ring (Ty), a structure derived from lower jaw elements. Middle ear bones are derived from the hyomandibular, and from both upper and lower jaw elements. (c) In diapsids, the position of the primary jaw joint did not move. Instead, the hyomandibular decoupled from the quadrate $(q)$ to form the columella (col).

Figure 2. Various ITD coding strategies.

(a) Gecko, left. Schematic drawing of the gecko brainstem. Auditory nerve (blue) conveys timing information to NM, which projects bilaterally to NL (purple). Modified from [36]. Right: ITD sensitive responses from left (purple) and right (blue) auditory nerve, best frequency $1 \mathrm{kHz}$. Large positive ITD values (blue) means sound maximally stimulates the ipsilateral (right) tympanic membrane, while ITD minima occur when sound reaches the contralateral (left) ear first and the coupling of the tympanic membranes suppresses right ear tympanic membrane vibration. Large negative ITD values (purple) curve were recorded from the left auditory nerve [34].

(b) Chicken, left: Schematic drawing of the chicken's brainstem. Axons from the ipsilateral NM enter NL dorsally, while those from contralateral NM enter ventrally to innervate the layer of NL neurons. Right: A range of ITD sensitive responses from $\mathrm{NL}$ cells tuned to $1 \mathrm{kHz}$ form a map of ITD in NL. Positive ITD values mean contralateral ear leading (i.e., sound arrives earlier at the contralateral ear than at the ipsilateral ear). 
(c) Gerbil, left: Schematic drawing of the gerbil's brainstem. Spherical bushy cells in the VCN (blue) provide excitatory inputs to the MSO, which also receives inhibitory inputs. Right: ITD response curves of MSO cells tuned at $1 \mathrm{kHz}$. In contrast to chicken's NL cells, the tuning curves of MSO neurons on each side of the brain are very similar. Peak positions of the tuning curves can lie out of the physiological ITD range. Modified from [40].

\section{References and recommended reading}

Papers of particular interest, published within the period of review, have been highlighted as:

* of special interest

** of outstanding interest

1. Wilczynski W: Central neural systems subserving a homoplasous periphery. Am Zool 1984, 24:755-763.

2. Carr CE, Christensen-Dalsgaard J: Sound Localization Strategies in Three Predators. Brain Behav Evol 2015, 86:17-27.

3. ${ }^{* *}$ Christensen CB, Christensen-Dalsgaard J, Madsen PT: Hearing of the African lungfish (Protopterus annectens) suggests underwater pressure detection and rudimentary aerial hearing in early tetrapods. J Exp Biol 2015, 218:381387.

A study of the African lungfish reveals a rudimentary ability to detect sound pressure. The finding expands our knowledge of how hearing may have evolved in early tetrapods, the first vertebrates to have limbs and digits.

4. Christensen-Dalsgaard J, Brandt C, Wilson M, Wahlberg M, Madsen PT: Hearing in the African lungfish (Protopterus annectens): pre-adaptation to pressure hearing in tetrapods? Biol. Lett. 2011, 7:139-141.

5. $\quad{ }^{*}$ Christensen CB, Lauridsen H, Christensen-Dalsgaard J, Pedersen M, Madsen PT: Better than fish on land? Hearing across metamorphosis in salamanders. Proc. R. Soc. B 2015, 282.

Salamanders can hear, despite not having an outer ear or tympanic middle ear, so early terrestrial vertebrates could probably hear as well.

6. ${ }^{* *}$ Clack JA: Evolutionary biology: The origin of terrestrial hearing. Nature 2015, 519:168-169.

This news and views focuses on the lungfish paper [4] but also provides a state of the art view of the underlying paleontology behind how hearing evolved in early tetrapods, the first vertebrates to have limbs and digits.

7. Christensen-Dalsgaard J, Carr CE: Evolution of a sensory novelty: tympanic ears and the associated neural processing. Br. Res. Bull. 2008, 75:365-370.

8. ${ }^{* *}$ Clack JA: Gaining ground: the origin and evolution of tetrapods. Indiana 
University Press; 2011.

Beginning with their closest relatives, the lobe-fin fishes such as lungfishes and coelacanths, Clack reviews the Devonian environment in which tetrapods evolved, defines tetrapods, describes their anatomy, and explains how they are related to other vertebrates.

9. $\quad * *$ Kitazawa T, Takechi M, Hirasawa T, Adachi N, Narboux-Nême N, Kume H, Maeda K, Hirai T, Miyagawa-Tomita S, Kurihara Y, et al.: Developmental genetic bases behind the independent origin of the tympanic membrane in mammals and diapsids. Nat Commun 2015, 6:6853.

This study showed that the eardrum and eustachian tubes evolved independently in mammals and diapsids. The mammalian eardrum depends on lower jaw formation, while that of diapsids develops from the upper jaw.

10. Manley GA: Some aspects of the evolution of hearing in vertebrates. Nature 1971, 230:506-509.

11. Northcutt RG, Popper A, Fay RR: Central Auditory Pathways in Anamniotic Vertebrates. 1980.

12. Northcutt RG: Ontogeny and phylogeny: a re-evaluation of conceptual relationships and some applications. Brain Behav Evol 1990, 36:116-140.

13. Modrell MS, Bemis WE, Northcutt RG, Davis MC, Baker CVH: Electrosensory ampullary organs are derived from lateral line placodes in bony fishes. Nat Commun 2011, 2:496-506.

14. McCormick CA: Central projections of the lateral line and eighth nerves in the bowfin, Amia calva. J Comp Neurol 1981, 197:1-15.

15. Edds-Walton PL, Fay RR, Highstein SM: Dendritic arbors and central projections of physiologically characterized auditory fibers from the saccule of the toadfish, Opsanus tau. J Comp Neurol 1999, 411:212-238.

16. ${ }^{* *}$ Edds-Walton PL: What the Toadfish Ear Tells the Toadfish Brain About Sound. Adv. Exp. Med. Biol. 2016, 877:197-226.

In fishes, binaural processes were believed to be unimportant due to the speed of sound in water and the acoustic transparency of the tissues in water. Combined anatomical and physiological recordings in brain show that binaural computations sharpen the directional information provided by the saccule.

17. Grothe B, Carr CE, Casseday JH, Fritzsch B, \& Köppl C: The evolution of central pathways and their neural processing patterns. In Evolution of the vertebrate auditory system, Edited by Manley GA, Popper AN \& Fay RR, Springer; 2004: 289-359.

18. Carr CE, Code RA: The central auditory system of reptiles and birds. In Comparative hearing: Birds and reptiles. Edited by Dooling RJ, Fay RR, Popper 
AN. Springer; 2000:197-248.

19. Yu W-M, Goodrich LV: Morphological and physiological development of auditory synapses. Hearing Research 2014, 311:3-16.

20. * Allen-Sharpley MR, Tjia M, Cramer KS: Differential roles for EphA and EphB signaling in segregation and patterning of central vestibulocochlear nerve projections. PLOS ONE 2013, 8:e78658.

Auditory and vestibular afferents enter the brainstem through the VIIIth cranial nerve and find targets in distinct brain regions. Ephrin mis-expression studies in VIllth nerve axons suggest axon guidance molecules contribute to formation of boundaries for patterning of inner ear projections in the hindbrain.

21. * Wullimann MF, Mueller T, Distel M, Babaryka A, Grothe B, Köster RW: The long adventurous journey of rhombic lip cells in jawed vertebrates: a comparative developmental analysis. Front. Neuroanat. 2011, 5:27.

This review summarizes the role of the vertebrate rhombic lip and its major derivatives. The authors propose that the rhombic lip evolved with jawed vertebrates.

22. Marín F, Puelles L: Morphological fate of rhombomeres in quail/chick chimeras: a segmental analysis of hindbrain nuclei. Eur J Neurosci 1995, 7:1714-1738.

23. Cambronero F, Puelles L: Rostrocaudal nuclear relationships in the avian medulla oblongata: A fate map with quail chick chimeras. J Comp Neurol 2000, 427:522-545.

24. Farago AF, Awatramani RB, Dymecki SM: Assembly of the brainstem cochlear nuclear complex is revealed by intersectional and subtractive genetic fate maps. Neuron 2006, 50:205-218.

25. Oertel D, Young ED: What's a cerebellar circuit doing in the auditory system? Trends in Neurosciences 2004, 27:104-110.

26. * Di Bonito M, Narita Y, Avallone B, Sequino L, Mancuso M, Andolfi G, Franzè AM, Puelles L, Rijli FM, Studer M: Assembly of the auditory circuitry by a Hox genetic network in the mouse brainstem. PLoS Genet. 2013, 9:e1003249.

This study on the regulation of Hox genes during development of the auditory system shows that auditory components derived from rhombomere 4 form functionally segregated sub-circuits in the developing auditory brainstem.

27. Willaredt, Schlüter T, Nothwang HG, Noth: The gene regulatory networks underlying formation of the auditory hindbrain. Experientia 2015, 72(3), 519535. http://doi.org/10.1007/s00018-014-1759-0

28. * Nothwang HG: Evolution of mammalian sound localization circuits: A developmental perspective. Prog. Neurob. 2016, 141:1-24. 
This review summarizes recent genetic analyses of auditory nuclei in fish, birds, and mammals, and discusses what is currently known about gene regulatory networks underlying development of auditory neurons across vertebrates.

29. Sienknecht UJ, Köppl C, Fritzsch B: Evolution and Development of Hair Cell Polarity and Efferent Function in the Inner Ear. Brain Behav Evol 2014, doi:10.1159/000357752.

30. Edds-Walton P, Fay RR: Directional and frequency response characteristics in the descending octaval nucleus of the toadfish (Opsanus tau). J. Comp physiol 2008, doi:10.1007/s00359-008-0373-8.

31. Jørgensen MB, Christensen-Dalsgaard J: Directionality of auditory nerve fiber responses to pure tone stimuli in the grassfrog, Rana temporaria. I. Spike rate responses. J. Comp physiol 1997, 180:493-502.

32. Christensen-Dalsgaard J, Manley GA: Acoustical coupling of lizard eardrums. JARO 2008, 9:407-416.

33. Vossen C, Christensen-Dalsgaard J, van Hemmen JL: Analytical model of internally coupled ears. JASA 2010, 128:909-918.

34. Christensen-Dalsgaard J, Tang Y-Z, Carr CE: Binaural processing by the gecko auditory periphery. J. Neurophys. 2011, 105:1992-2004.

35. Zeddies DG, Fay RR, Gray MD, Alderks PW, Acob A, Sisneros JA: Local acoustic particle motion guides sound-source localization behavior in the plainfin midshipman fish, Porichthys notatus. J Exp Biol 2011, 215:152-160.

36. Tang $Y-Z$, Christensen-Dalsgaard J, Carr CE: Organization of the auditory brainstem in a lizard, Gekko gecko. I. Auditory nerve, cochlear nuclei, and superior olivary nuclei. J Comp Neurol 2012, 520:1784-1799.

37. *Manley GA: Evolutionary Paths to Mammalian Cochleae. JARO 2012, 13:733-743.

Three parallel developments over $150 \mathrm{Ma}$ led to high-frequency hearing only in most modern therian cochleae. These include lighter middle bones, an elongated cochlea and more effective prestin motors.

38. Kettler L, Christensen-Dalsgaard J, Larsen ON, Wagner H: Low frequency eardrum directionality in the barn owl induced by sound transmission through the interaural canal. Biol Cyber 2016, doi:10.1007/s00422-016-0689-3.

39. Tollin DJ, Koka K: Linear and Nonlinear Coding of Sound Spectra by Discharge Rate in Neurons Comprising the Ascending Pathway Through the Lateral Superior Olive. In: The Neurophysiological Bases of Auditory Perception, 2010. 143-153.

40. Ashida G, Carr CE: Sound localization: Jeffress and beyond. Curr Opin Neurobiol 2011, doi:10.1016/j.conb.2011.05.008. 
41. Carr CE, Shah S, McColgan T, Ashida G, Kuokkanen PT, Brill S, Kempter R, Wagner $\mathrm{H}$ : Maps of interaural delay in the owl's nucleus laminaris. $J$. Neurophys. 2015, 114:1862-1873.

42. ** Palanca-Castan N, Köppl C: In vivo Recordings from Low-Frequency Nucleus Laminaris in the Barn Owl. Brain Behav Evol 2015, 85:271-286.

Recordings from low-frequency NL in the barn owl are consistent with a delay line model, so differences between ITD processing systems are more likely to have originated through divergent evolution of different vertebrate groups.

43. Köppl C, Carr CE: Maps of interaural time difference in the chicken's brainstem nucleus laminaris. Biol Cyber 2008, 98:541-559.

44. * Palanca-Castan N, Köppl C: Change in the coding of interaural time difference along the tonotopic axis of the chicken nucleus laminaris. Front. Neural Circuits 2015, 9:43.

In vivo recordings from chicken NL support the presence of modified maps of best ITD, that allow the chicken to use the slopes of ITD-selective responses instead of their peaks to topographically represent ITD at mid- to high frequencies.

45. McAlpine D, Jiang D, Palmer AR: A neural code for low-frequency sound localization in mammals. Nat Neurosci 2001, 4:396-401.

46. * Harper NS, Scott BH, Semple MN, McAlpine D: The neural code for auditory space depends on sound frequency and head size in an optimal manner. PLOS ONE 2014, 9:e108154.

The optimal-coding model predicts that distributions of best ITDs depend on head size and sound frequency: for high frequencies and large heads it resembles the classic model; for low frequencies and small head sizes it resembles the bimodal model. Reanalysis of a large pool of data suggests different representations are used at different sound frequencies.

47. Striedter GF: Stepping into the same river twice: homologues as recurring attractors in epigenetic landscapes. Brain Behav Evol 1998, 52:218-231.

48. ${ }^{* *}$ Grothe B, Pecka M: The natural history of sound localization in mammals a story of neuronal inhibition. Front. Neural Circuits 2014, 8:116.

This comparison of neuronal mechanisms for sound localization in mammals and other tetrapods highlights the independent origins of tympanic hearing, and suggests that mammalian hearing evolved initially mainly to process ILD, and that the ITD processing in mammals is derived from ILD processing.

49. Heffner RS, Heffner HE: Evolution of sound localization in mammals. In: The evolutionary biology of hearing. Edited by Fay RR, Popper AN, and Webster DB. Springer-Verlag, 1992:691-715.

50. De Beer GR: The vertebrate skull. Oxford University Press, 1937. 


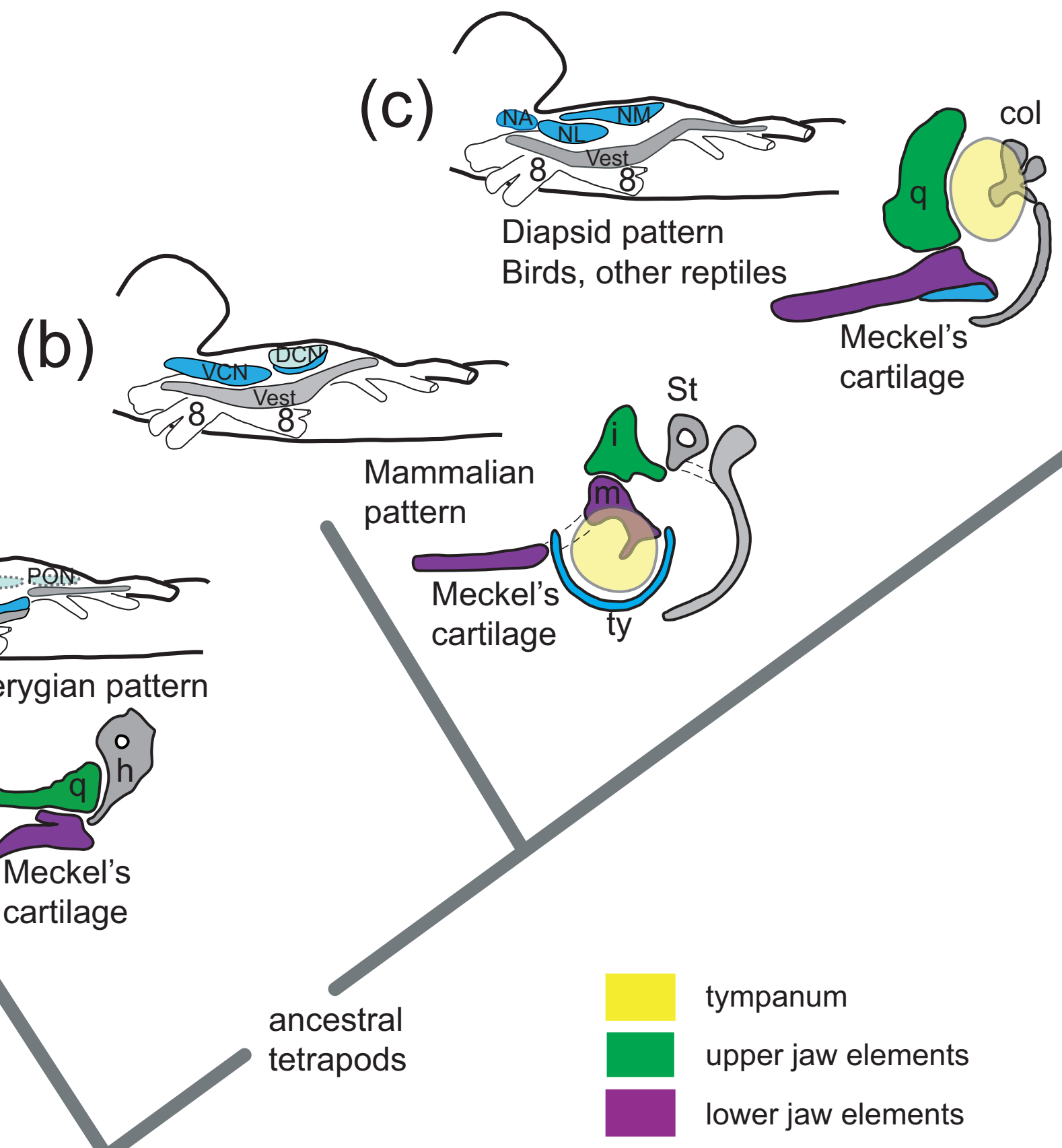

Figure 1. Carr and Christensen-Dalsgaard

(a)

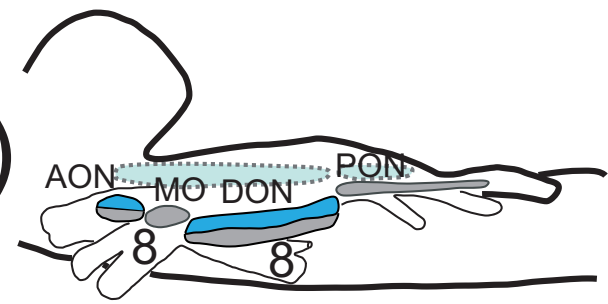

Actinopterygian pattern

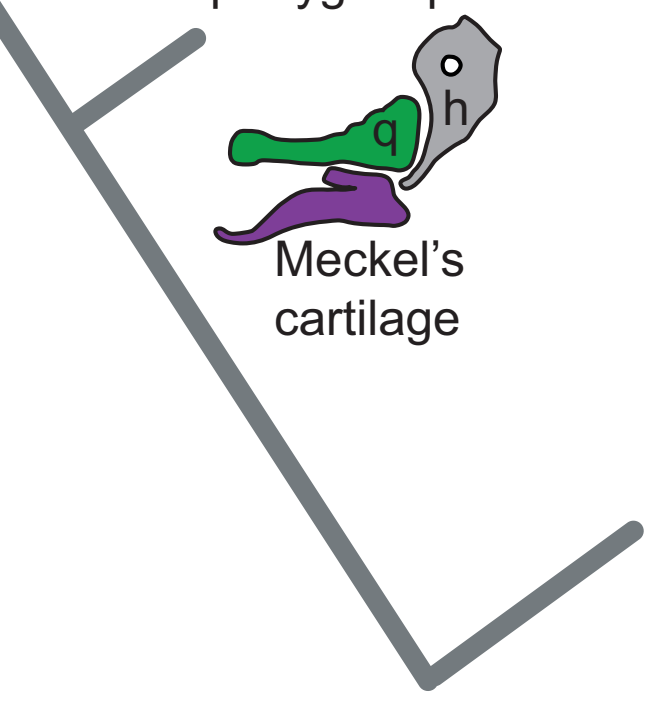

ancestral

tetrapods lower jaw elements 
("are)

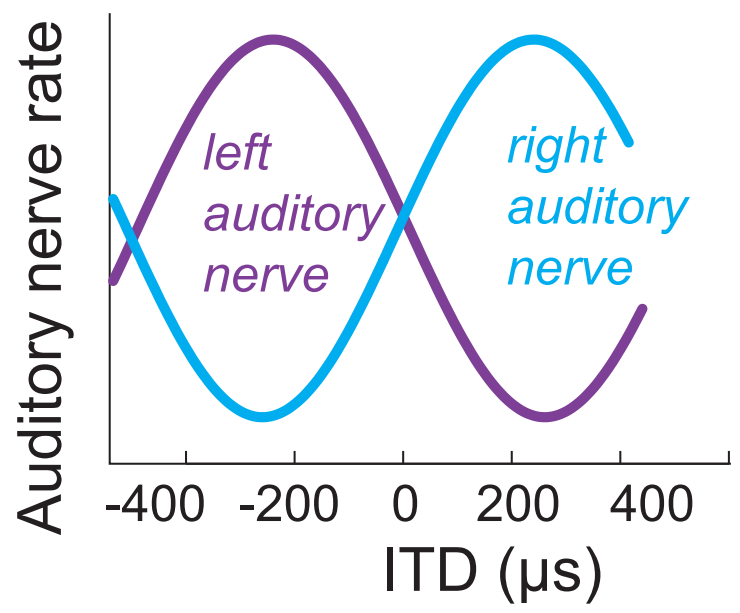

(b)

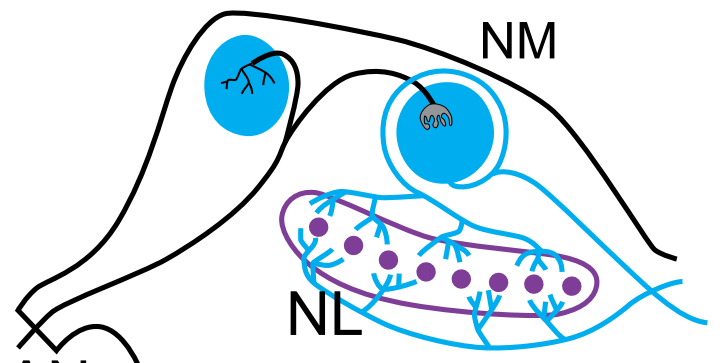

AN

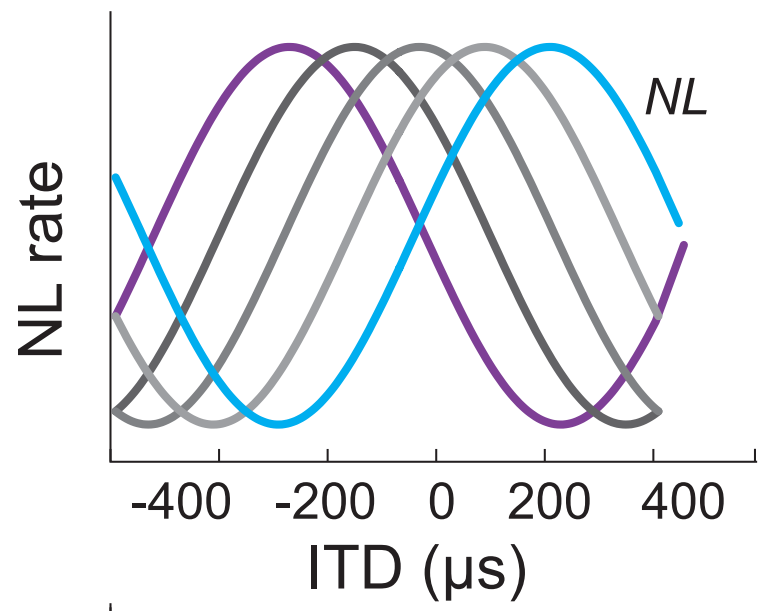

(c)
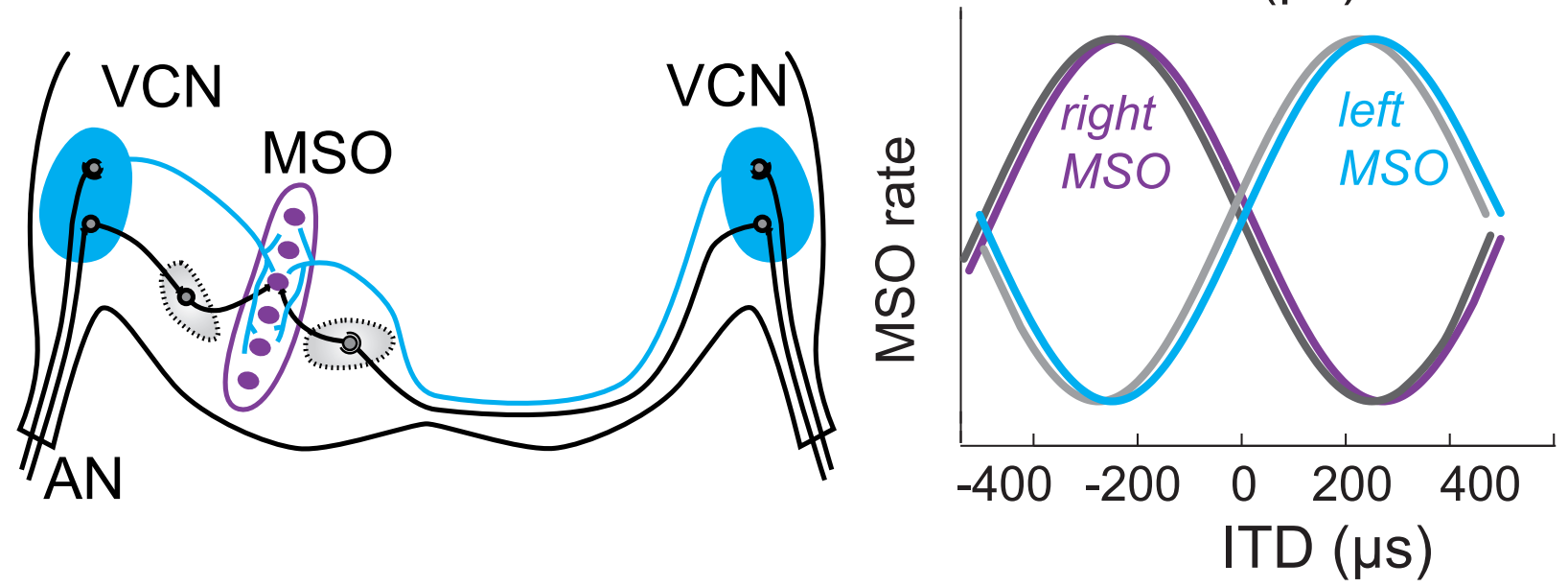

Figure 2. Carr and Christensen-Dalsgaard 